\title{
A questionnaire study investigating the prevalence of chronic postoperative pain
}

\author{
Sung-Hwan Cho, Young-Rok Kim, Joon-Ho Lee, Sang-Hyun Kim, Won-Seok Chae, Hee-Cheol Jin, \\ Jeong-Seok Lee, and Yong-Ik Kim \\ Department of Anesthesiology and Pain Medicine, Soonchunhyang University Bucheon Hospital, Bucheon, Korea
}

Background: Chronic postoperative pain (CPOP) is defined as pain of at least 2 months duration after a surgical procedure. Until recently, it has been a neglected topic, because it can occur after a wide spectrum of operations; however, little is known regarding its underlying mechanism, prevalence, risk factors, and treatments. We investigated characteristics of CPOP after various operations via a questionnaire.

Methods: Patients were contacted at $>2$ months after surgery, irrespective of sex, type of operation or anesthesia method, and a follow-up pain questionnaire was administered by phone.

Results: One hundred forty-five of 400 patients (36.3\%) described CPOP. The prevalence of CPOP was significantly lower in laparoscopic surgery $(29 / 159,18.2 \%)$ than open surgery $(116 / 241,48.1 \%)$. The prevalence of CPOP was higher with the use of PCA (patient controlled analgesia), (45.3\%) than without PCA (24.6\%). There were no significant differences regarding sex, anesthetic method, or duration of operation.

Conclusions: Our results indicate that the prevalence of CPOP may be related to use of an endoscope and PCA. However, it is difficult to completely explain the correlation, because this is a complex area of research. More research is needed to improve the quality of pain relief. (Korean J Anesthesiol 2012; 62: 40-46)

Key Words: Chronic postoperative pain, Prevalence, Questionnaire.

Received: March 3, 2011. Revised: June 7, 2011. Accepted: July 18, 2011.

Corresponding author: Sung-Hwan Cho, M.D., Department of Anesthesiology and Pain Medicine, Soonchunhyang University Bucheon Hospital, 1174, Jung-dong, Wonmi-gu, Bucheon 420-767, Korea. Tel: 82-32-621-5330, Fax: 82-32-621-5322, E-mail: singaring@schmc.co.kr (c) This is an open-access article distributed under the terms of the Creative Commons Attribution Non-Commercial License (http:// creativecommons.org/licenses/by-nc/3.0/), which permits unrestricted non-commercial use, distribution, and reproduction in any medium, provided the original work is properly cited. 


\section{Introduction}

Chronic postoperative pain or persistent post-surgical pain have been the terms used to explain continuous pain symptoms that occur after surgery. Since Crombie et al. [1] used this terminology in their paper, a 'correct' definition still has not agreed upon. In this survey, chronic postoperative pain is defined as pain that continues for $>2$ months after an operation, which was also the definition used in a study on chronic postoperative pain by Macrae [2]. Patients are very anxious of even short-term pain after surgery, and there could be adverse physiological effects according to the degree of pain. If this type of pain continues, the patient suffers physical and psychological problems as well as negative consequences in terms of social adaptation, and increased medical costs. Chronic postoperative pain can occur regardless of the type of operation, and recently, interest in this area has increased. There have been several individual studies conducted on chronic postoperative pain for various types of surgery such as mastectomy, thoracotomy, and laparoscopic gynecological surgery [3-6]. However, there are few reports regarding the occurrence rate of chronic postoperative pain according to age and sex, the type of operation, risk factors that cause chronic postoperative pain, and regarding the results of using PCA. Therefore, the aim of this study was to investigate the frequency of chronic postoperative pain in various surgeries conducted in 5 departments, including general surgery, chest surgery, OB \& GYN, orthopedics, and urology, and the factors that affect the occurrence of chronic postoperative pain.

\section{Materials and Methods}

Patients aged $>20$ years but $<70$ years who underwent scheduled surgery at our hospital two months earlier were randomly contacted to conduct a telephone questionnaire survey regardless of the type of anesthesia, operation or use of endoscopy. Those who refused to participate in the telephone questionnaire survey and patients who found it difficult to complete the questionnaire over the phone were excluded from the study, as well as patients who underwent emergency surgery or had underlying illnesses such as chronic liver disorder, diabetes, or renal insufficiency. The number of patients who completed the questionnaire was 400 (133 males and 267 females).

Before completing the questionnaire, the purpose of the study was explained to the subjects and their participation was requested. The questionnaire was given by a pain coordinator nurse only to subjects who gave their consent and required 2025 minutes to complete.

The patients' sex, age, type of operation and the time it required, anesthesia method, use of endoscopy, and use of PCA (patient controlled analgesia) were surveyed. In addition, when there was pain, the area of pain and its condition was asked and recorded. When the patient could not remember the method of anesthesia or operation and the method of pain control, the anesthetic records of the patient were used for reference. The degree of pain was measured with the Verbal Numerical Rating Scale (VNRS, 0-10), and it was checked whether the pain inconvenienced daily life. In addition, whether there was any psychological stress and additional treatment along with the type of treatment the patient received was investigated (Appendix 1).

SPSS 12.0 (SPSS Inc. Chicago, USA) was used for statistical analysis. The variables for age, sex, operative time, use of endoscope, anesthetic method, and PCA were tested with the chi-square test, and the results were considered statistically significant when the $\mathrm{P}$ value was $<0.05$.

\section{Results}

From the patients who responded to the questionnaire, 42 males and 103 females complained of pain. The age distribution was from a minimum 20 to a maximum 68 , and the mean age was 40.1. Distribution was highest in the $41-50$ age group with 140 subjects (35\%), and the highest number of patients who complained of pain was in the $61-70$ age group (60\%) (Table 1 ).

Regarding anesthesia, 372 patients underwent general anesthesia and 28 patients underwent local anesthesia. Chronic postoperative pain occurred in 134 patients who underwent general anesthesia and in 11 patients who underwent local anesthesia.

Distribution of operation type and operative time were as follows. From 5 surgical departments (general surgery, chest surgery, OB \& GYN, orthopedics, and urology), 10 out of 17 patients $(58.8 \%)$ who received chest surgery complained of pain, with the highest rate of occurrence, and next, were the patients who received OB \& GYN surgery with 62 out of 141 patients $(44.0 \%$ ) (Table 2). In the distribution of the operative

Table 1. Demographic Data

\begin{tabular}{ccc}
\hline Variable & $\begin{array}{c}\text { No Pain } \\
(\mathrm{N}=255)\end{array}$ & $\begin{array}{c}\text { Pain } \\
(\mathrm{N}=145)\end{array}$ \\
\hline Sex & & \\
$\mathrm{M}(\mathrm{N}=133)$ & $91(68.4 \%)$ & $42(31.6 \%)$ \\
$\mathrm{F}(\mathrm{N}=267)$ & $164(61.4 \%)$ & $103(38.6 \%)$ \\
Age $(\mathrm{yr})$ & & \\
$20-30(\mathrm{~N}=71)$ & $45(63.4 \%)$ & $26(36.6 \%)$ \\
$31-40(\mathrm{~N}=116)$ & $78(67.2 \%)$ & $38(32.8 \%)$ \\
$41-50(\mathrm{~N}=140)$ & $90(64.3 \%)$ & $50(35.7 \%)$ \\
$51-60(\mathrm{~N}=63)$ & $38(60.3 \%)$ & $25(39.7 \%)$ \\
$61-70(\mathrm{~N}=10)$ & $4(40 \%)$ & $6(60 \%)$ \\
\hline
\end{tabular}

Values are number of patients (\%). 
Table 2. Characteristics of Patients with and without CPOP

\begin{tabular}{lcc}
\hline \multicolumn{1}{c}{ Variable } & $\begin{array}{c}\text { No Pain } \\
(\mathrm{N}=255)\end{array}$ & $\begin{array}{c}\text { Pain } \\
(\mathrm{N}=145)\end{array}$ \\
\hline Use of endoscope & & \\
Yes $(\mathrm{N}=159)$ & $130(81.8 \%)$ & $29(18.2 \%)$ \\
No $(\mathrm{N}=241)$ & $125(51.9 \%)$ & $116(48.1 \%)$ \\
Type of anesthesia & & \\
General $(\mathrm{N}=372)$ & $238(60.7 \%)$ & $134(39.3 \%)$ \\
Regional $(\mathrm{N}=28)$ & $17(63.7 \%)$ & $11(36.3 \%)$ \\
Operation time $(\mathrm{hr})$ & & \\
$0-1(\mathrm{~N}=51)$ & $27(52.9 \%)$ & $24(47.1 \%)$ \\
$1-2(\mathrm{~N}=182)$ & $133(73.1 \%)$ & $49(26.9 \%)$ \\
$2-3(\mathrm{~N}=81)$ & $51(63.0 \%)$ & $30(37.0 \%)$ \\
$3-4(\mathrm{~N}=33)$ & $13(39.4 \%)$ & $20(60.6 \%)$ \\
$>4(\mathrm{~N}=53)$ & $31(58.5 \%)$ & $22(41.5 \%)$ \\
Departments of surgery & & \\
General surgery $(\mathrm{N}=201)$ & $141(70.2 \%)$ & $60(29.8 \%)$ \\
Chest surgery $(\mathrm{N}=17)$ & $7(41.2 \%)$ & $10(58.8 \%)$ \\
OB \& GYN $(\mathrm{N}=141)$ & $79(56.0 \%)$ & $62(44.0 \%)$ \\
Orthopedics $(\mathrm{N}=25)$ & $15(60 \%)$ & $10(40 \%)$ \\
Urology $(\mathrm{N}=16)$ & $13(81.2 \%)$ & $3(18.8 \%)$ \\
PCA & & \\
Intravenous $(\mathrm{N}=195)$ & $109(55.9 \%)$ & $86(44.1 \%)$ \\
Epidural $(\mathrm{N}=30)$ & $14(46.7 \%)$ & $16(53.3 \%)$ \\
No use $(\mathrm{N}=175)$ & $132(75.4 \%)$ & $43(24.6 \%)$ \\
\hline
\end{tabular}

Values are number of patients (\%). OB \& GYN: obstetric and gynecologic,chronic postoperative pain.

time, 20 out of 33 patients (60.6\%) who received operations of $3-4$ hours had the highest occurrence rate of pain.

A total of 159 operations used endoscopes, including laparoscopes, and from these, 29 patients (18.2\%) complained of pain, so the rate of pain occurrence was noticeably lower than when endoscopes were not used (48.1\%).

In the distribution of PCA use, out of the 225 patients who had PCA, 195 had intravenous PCA while 30 had epidural PCA. From these, 86 patients (44.1\%) and 16 patients (53.3\%), respectively, complained of pain; thus, it was higher than in cases with no PCA use.

From patients who experienced pain, the distribution of VNRS was highest for a pain scale score of 2, with 38 patients, then a pain scale score of 4 , with 32 patients, and finally, a pain scale score of 3 , with 26 patients (Fig. 1).

For the area that patients complained of for pain, 106 patients responded that the pain was limited to the operated area, 19 responded that the pain expanded from the operated area to the surrounding area, and 20 complained of pain in areas unrelated to the operation. For the condition of pain, 8 patients complained of allodynia, 1 of referred pain, 6 of numbness, and 28 patients complained of a combination of $>2$ types of pain. Other than this, 64 patients responded they still experienced pain even when remaining still, and 24 patients responded they experienced pain when coughing or moving. Thirteen patients responded that psychological stress was disrupting everyday

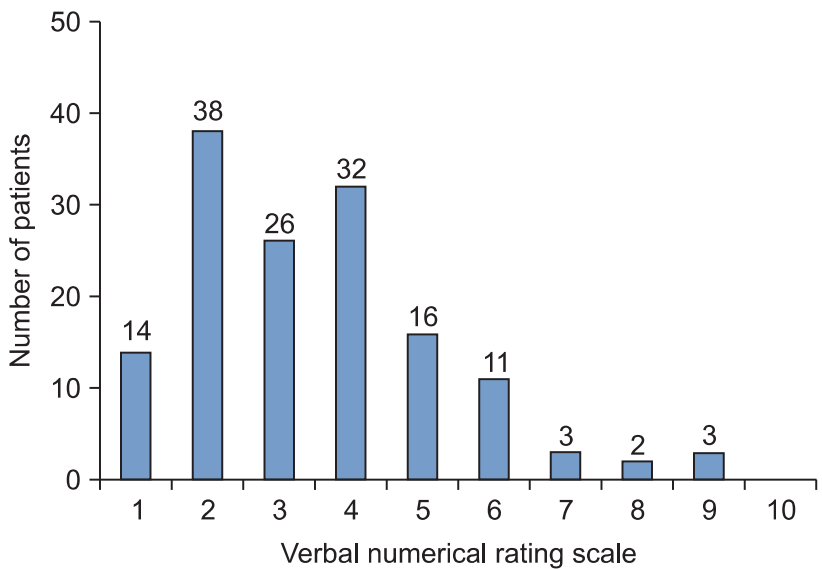

Fig. 1. This graph shows the distribution of verbal numercial rating scale in 145 patients with chronic post-operative pain.

life, and in severe cases, 2 patients expressed that they wanted to amputate the nerve. Additional treatment consisted of taking painkillers, physical therapy, pain relief patch, etc.

\section{Discussion}

Patients and even surgeons who perform the operations are afraid of pain that continues after the operation. Not only is there the primary problem of extended hospital stays and increased medical costs, but also pain can become a severe hindrance to returning to everyday life and can hinder the social adaptation of the patient for a long time, as in failure syndrome after spinal surgery [7]. Recently, in the pain clinic at our hospital, there was an increase in patients visiting for postoperative pain from various age groups. Complaints of pain were found to be from operations with long operative time and large incisions such as spinal or colon cancer surgery, but also in simple surgeries such as the removal of small nodules or hernia. Despite the increase in patients suffering from postoperative pain, until now, it was difficult to find research conducted in Korea regarding chronic postoperative pain reported in the literature. In previously published literature, it was limited to a certain type of surgery such as a comparison of analgesic requirements after orthopedic surgery [8], or limited to studies on reducing acute pain shortly after surgery through specific procedures or administration of medication [9]. In other countries, there have been many studies conducted to find explanations for pain caused by surgery that did not resolve itself or was cured after a certain period of time. Especially, Macrae [2] first reported on chronic postoperative pain in 2001 through a statistical method using a meta-analysis of $\sim 400$ different reports. 10 years of additional research, Macrae reported on factors that cause chronic postoperative pain and preventive measures [10], and in this report, race, 
Table 3. Predictive Factors of Chronic Postoperative Pain

\begin{tabular}{lccc}
\hline \multicolumn{1}{c}{ Variable } & Odds ratio & P value & 95\% Confidence interval \\
\hline Sex & 1.23 & 0.421 & $0.74-2.03$ \\
Age & 1.01 & 0.348 & $0.99-1.03$ \\
Use of endoscope & 0.27 & $0.000^{*}$ & $0.16-0.43$ \\
Type of anesthesia & 0.86 & 0.730 & $0.37-1.99$ \\
Operation time & 1.00 & 0.862 & $0.99-1.00$ \\
PCA & 1.74 & $0.005^{*}$ & $1.18-2.56$ \\
\hline
\end{tabular}

PCA: patient controlled analgesia. *Statistically significant, $\mathrm{P}<0.05$.

age, sex, social and environmental factors, genetic factors, conditions before surgery, surgical factors, anesthesia, and pain relief were reported to be causal factors. Hinrichs-Rocker et al. [11] reported a connection between socio-psychological factors such as depression, stress, education level, and racial differences with chronic postoperative pain, while Kehlet et al. [12] reported that genetics, the existence of preceding pain, psychosocial factors, age, and sex were factors that caused chronic postoperative pain. Our study referred to the reported literature and we centered our investigation on age, sex, operative time, anesthetic method, endoscopy, and use of PCA as factors that could cause chronic postoperative pain (Table 3).

Even now, the mechanism for chronic postoperative pain is not precisely known, and this is because various pain syndromes can be caused by different mechanisms even after the same surgery. This makes it difficult to simply explain the cause of chronic postoperative pain. Generally, damage to nerve tissues from the surgery and neuronal plasticity are considered to be the cause of this kind of chronic postoperative pain $[10,12]$.

Many chronic pain symptoms after surgery such as complex regional pain syndrome, phantom limb pain, post-thoracotomy pain, and post-mastectomy pain syndrome are all thought to be related to neurological symptoms [1].

Even in our study, patients complained of numbness, allodynia, or pain in areas unrelated to the operated area, which seems to indicate an association with neurological symptoms.

In this study, the number of patients who experienced postoperative pain was $145(36.3 \%)$. In previous studies [10,12], the occurrence rate of chronic postoperative pain showed a relatively wide range of $10-50 \%$, and exhibited a difference according to the type of operation; for example, mastectomy was $20-50 \%$, caesarean section $6 \%$, amputation $50-85 \%$, and thoracotomy $5-65 \%$. Our study included 5 different surgical departments, and the occurrence rates for pain were $29.8 \%$ for general surgery, $40.0 \%$ for orthopedics, $58.8 \%$ for chest surgery, $44.0 \%$ for OB \& GYN, and $18.8 \%$ for urology.

The degree of pain can be different according to operated area and operation time, and this could be because the range or degree of damage during the operation can be intensified. In a report that investigated the frequency of use of pain control methods in different surgical departments [13], a high frequency was shown in the order of orthopedics, general surgery, internal medicine, and OB \& GYN; and from these, PCA was used the most in musculoskeletal operations. Our study showed an occurrence rate in the order of chest surgery, OB \& GYN, orthopedics, general surgery, and urology. This is possibly because it is generally easy to damage intercostals nerves in thoracotomy due to the anatomical structure. However, in recent chest surgery, there has been an increase in the endoscope approach, which reduces this risk. In the reference materials that were organized and the presented research regarding the occurrence of pain after thoracotomy $[2,14,15]$, there were various results on pain occurrence after thoracotomy, but clearly, general thoracotomy had significant long-term danger, while thoracoscopic surgery reduced this occurrence of pain. In this aspect, the relationship between the operative time and the occurrence of chronic pain seems to be proportionate, and thus, the occurrence rate should be highest in surgeries of $>4$ hours, but our research results showed that the occurrence rate was highest in surgeries of 3-4 hours with $60.6 \%$, and was not proportionate to the operative time. However, as the operative time increased, the occurrence of severe pain led to high amounts of morphine use [16]. In addition, there are reports of many occurrences of chronic pain or other complications in surgeries of $>3$ hours [17] so there is a need to expand the study population or investigate using other methods regarding these results.

In the distribution according to the $61-70$ age group, the group had a $60 \%$ occurrence, but there were no significant differences in the other age groups. Seo et al. [18] reported that in their analysis of patients from age 10-80 who visited their pain clinic, the 60-69 age group accounted for the largest group who visited the clinic $(29.8 \%)$ while patients between the ages of $40-69$ accounted for $69.2 \%$ of the total. In similar reports, patients in the $60-69$ age group accounted for $18.6 \%$ of the total number of patients who visited the pain clinic [19], and this is thought to be because as age increases, the possibility of neurological diseases also increases [20]. Our research results cannot be seen as identical to the general chronic pain patient group, but our results were similar, so it is considered that chronic postoperative pain has a certain degree of association with old age.

A peculiarity of our study was that it had different results compared to other reports regarding the use of PCA [21], where $44.1 \%$ of intravenous PCA and $53.3 \%$ of epidural PCA had chronic postoperative pain, which was higher than when PCA was not administered. These results should not be interpreted to mean that PCA is a cause of pain, but should be interpreted as related to another problem, such as an error in patient selection. In this survey, the number of patients who underwent 
an operation for $>2$ hours was 181, and from these, 131 patients requested PCA, and especially, from 97 patients who underwent an operation for $>3$ hours, 84 requested PCA. For these reasons, the error was thought to be caused by PCA use being concentrated in the high-risk group rather than in a comparison of similar groups. In another study, [13] PCA use was highest in patients who underwent musculoskeletal surgery and then in OB \& GYN, which shows that PCA use usually occurs in cases where severe pain is anticipated, whereas PCA was not used in relatively simple and short operations.

Surgeries that utilize endoscopes have been conducted in various areas such as urology, general surgery, and OB \& GYN, and recently in thyroidectomy [22]. Endoscopic surgery has many advantages such as a reduction in operative scars, fast recovery, and reduced postoperative pain; thus, many patients and medical professionals prefer it. In our study, the number of patients who underwent endoscopic surgery and experienced pain was $18.2 \%$, which was lower than in the nonendoscopic surgery (48.1\%), and this is thought to be because the incision is relatively smaller and damage to nerve and tissue during the operation is reduced. This reduces the occurrence of neuronal plasticity, which as a result reduces the occurrence of chronic pain [12]. From endoscopic surgery, those that used a laparoscope had less nerve damage compared to a laparotomy and thus, showed significantly lower occurrence of pain. However, caution is required in endoscopic surgery because of the inconvenient posture of the patient and pressed nerves due to excessive pressure from gas [23].

There are reports that general anesthesia is associated more with severe pain after surgery compared to local anesthesia [10], but use of medication can be different according to the anesthesia method so, to a certain extent, judging anesthesia method simply as an independent variable is debatable. In our study, there were no significant differences in the association of pain occurrence with anesthesia method.

This study was conducted to investigate the association between surgery and chronic pain, in other words, causal factors, but there were limitations and insufficient areas to accurately reflect the characteristics that are connected to the occurrence of chronic postoperative pain. Firstone limitation of this study was that it was done over the telephone, so the information that was collected was according to the patients' own answers, and subjective factors could not be excluded. Next, there was not enough information collected on the patients before the operation, so environmental factors such as occupation, education, and a history of drinking and smoking could not be included. In addition, for the use of PCA, which had unexpected results in our study, a control study that excludes other factors that affect the occurrence of chronic postoperative pain should be conducted. Recently, chronic postoperative pain has been on the rise in Korea, but there are not much data yet, and statistical analyses have not been done so we hope that more interest and various efforts will continue to help understand the characteristics of chronic postoperative pain.

In conclusion, from the factors surveyed in this study, endoscopic surgery and use of PCA were significant factors related to the occurrence rate of chronic postoperative pain. Other factors such as age, sex, method of operation, and anesthesia were associated with the occurrence of chronic postoperative pain, but were not statistically significant.

\section{References}

1. Crombie IK, Davies HT, Macrae WA. Cut and thrust: antecedent surgery and trauma among patients attending a chronic pain clinic. Pain 1998; 76: 167-71.

2. Macrae WA. Chronic pain after surgery. Br J Anaesth 2001; 87: 8898.

3. Hazelrigg SR, Cetindag IB, Fullerton J. Acute and chronic pain syndromes after thoracic surgery. Surg Clin North Am 2002; 82: 849-65.

4. Stevens PE, Dibble SL, Miaskowski C. Prevalence, characteristics, and impact of post mastectomy pain syndrome: an investigation of women's experiences. Pain 1995; 61: 61-8.

5. Wallace MS, Wallace AM, Lee J, Dobke MK. Pain after breast surgery: a survey of 282 women. Pain 1996; 66: 195-205.

6. Katz J, Cohen L. Preventive analgesia is associated with reduced pain disability 3 weeks but not 6 months after major gynecologic surgery by laparotomy. Anesthesiology 2004; 101: 169-74.

7. Thomson S, Jacques L. Demographic characteristics of patients with severe neuropathic pain secondary to failed back surgery syndrome. Pain Pract 2009; 9: 206-15.

8. Hwang SH, Kim HH, Shin JW, Leem CG, Lee C, Yang HS, et al. Comparison of analgesic requirements for postoperative pain control in patients undergoing major orthopedic surgery. J Korean Pain Soc 2004; 17: 228-33.

9. Lee C, Kim TY. The effect of preoperative dexamethasone administration, according to age and gender on postoperative pain in patients who undergo laparoscopic cholecystectomy. Korean J Pain 2008; $21: 51-6$.

10. Macrae WA. Chronic post-surgical pain: 10 years on. Br J Anaesth 2008; 101: 77-86.

11. Hinrichs-Rocker A, Schulz K, Järvinen I, Lefering R, Simanski C, Neugebauer EA. Psychosocial predictors and correlates for chronic post-surgical pain (CPSP) - a systematic review. Eur J Pain 2009; 13: 719-30.

12. Kehlet H, Jensen TS, Woolf CJ. Persistent postsurgical pain: risk factors and prevention. Lancet 2006; 367: 1618-25.

13. Kim JH, Lee YH, Yang BM. A national survey of postoperative pain managements in hospitals from the national health insurance database. Korean J Anesthesiol 2008; 55: 458-66.

14. Perttunen K, Tasmuth T, Kalso E. Chronic pain after thoracic surgery: a follow-up study. Acta Anaesthesiol Scand 1999; 43: 563-7. 
15. Pluijms WA, Steegers MA, Verhagen AF, Scheffer GJ, Wilder-Smith $\mathrm{OH}$. Chronic post-thoracotomy pain: a retrospective study. Acta Anaesthesiol Scand 2006; 50: 804-8.

16. Dahmani S, Dupont H, Mantz J, Desmonts JM, Keita H. Predictive factors of early morphine requirements in the post anaesthesia care unit (PACU). Br J Anaesth 2001; 87: 385-9.

17. Peters ML, Sommer M, de Rijke JM, Kessels F, Heineman E, Patijn J, et al. Somatic and psychologic predictors of long-term unfavorable outcome after surgical intervention. Ann Surg 2007; 245: 487-94.

18. Seo CM, Mun CW, Baek UY, Hong JG. A survey of patients visiting pain clinic. J Korean Pain Soc 2000; 13: 224-31.

19. Park EJ, Han KR, Kim DW, Kim C. A clinical survey of the patients in neuro-pain clinic at Ajou university. Korean J Pain 2007; 20: 181-5.
20. Jung BF, Johnson RW, Griffin DR, Dworkin RH. Risk factors for postherpetic neuralgia in patients with herpes zoster. Neurology 2004; 62: 1545-51.

21. Momeni M, Crucitti M, De Kock M. Patient-controlled analgesia in the management of postoperative pain. Drugs 2006; 66: 2321-37.

22. Jeong JJ, Kang SW, Yun JS, Sung TY, Lee SC, Lee YS, et al. Comparative study of endoscopic thyroidectomy versus conventional open thyroidectomy in papillary thyroid microcarcinoma (PTMC) patients. J Surg Oncol 2009; 100: 477-80.

23. Choy YK, Kim MH, Jo DH, Kim IH. Ilioinguinal and iliohypogastric nerve block for neuropathic pain following the laparoscopic surgery - A case report. J Korean Pain Soc 1998; 11: 124-6. 
Appendix 1. Questionnaire for Chronic Postoperative Pain

"Hello. We are calling from 00 hospital pain clinic. Two months have passed since your surgery. We have called to find out whether you are currently experiencing any pain or have any other reasons for discomfort."

(Tel. 000 - 0000)

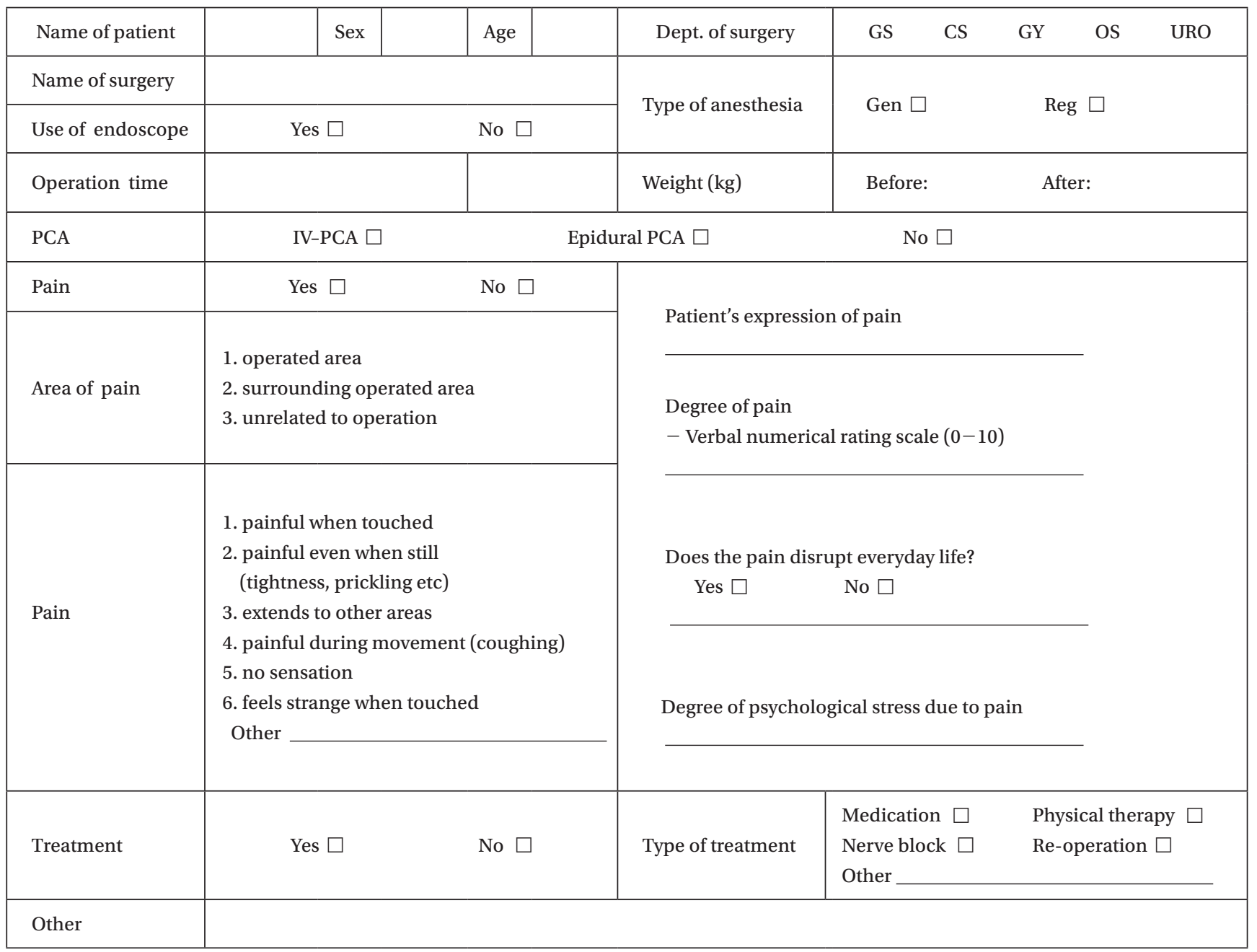

